\begin{tabular}{l|l|l|l|l|l} 
Revista Praxis & ISSN: $1657-4915$ & Vol. 13 & No. 1 & $56-68$ & enero - junio de 2017
\end{tabular}

DOI: http://dx.doi.org/10.21676/23897856.2068

\title{
LOS GRUPOS INFORMALES EN LAS ORGANIZACIONES ESCOLARES: UN ACERCAMIENTO DESDE LA GESTIÓN EDUCATIVA
}

\author{
INFORMAL GROUPS IN SCHOOL ORGANIZATIONS: AN APPROACH THROUGH \\ EDUCATIONAL MANAGEMENT
}

\author{
Jorge Oswaldo Sánchez-Buitrago $^{(i D}$, Javier Viloria-Escobar ${ }^{2}{ }^{(D}$ y Luis Francisco Miranda-Terraza ${ }^{3}$
}

\begin{abstract}
RESUMEN
El presente trabajo pretende caracterizar los grupos informales existentes en las instituciones escolares de orden público en el distrito de Santa Marta, así como develar los factores que originan tales agrupaciones y sus influencias en la calidad educativa; por lo cual, prioriza una metodología de corte etnográfico, fundamentada en la perspectiva de la complementariedad, que hace uso de instrumentos como: análisis documental, diarios de campo, observación participante y entrevistas en profundidad para comprender la realidad social de las organizaciones educativas. En los resultados preliminares se evidencian diversos grupos informales al interior de las estructuras legales, grupos que se originan por factores como intereses comunes, relaciones de amistad, las posiciones ideológicas y prácticas de actividades deportivas y culturales. La conclusión central del estudio enuncia unas posibles relaciones entre la existencia los grupos y la calidad educativa.
\end{abstract}

Palabras clave: Grupos informales; relaciones sociales; organizaciones escolares; influencias en las organizaciones; calidad educativa.

\section{ABSTRACT}

This paper aims to characterize the informal groups existing in public schools in the district of Santa Marta (Colombia), as well as to unveil the factors which cause such groups to exist. It aims too at analyzing their influences on educational quality. Methodologically-wise, the study builds upon an ethnographic approach, based on the perspective of complementary instruments, such as: documentary analysis, field diaries, participant observation and in-depth interviews to understand the social reality of educational organizations. As preliminary findings, several informal groups are found to exist within the legal structures, groups that result from factors such as common interests, friendship, shared ideological positions and sports and cultural practices. The main conclusion of this study is that there may be relationships between the presence of the groups and educational quality.

Keywords: Informal groups; social relationships; school organizations; influences on organizations; educational quality.

Descriptores JEL I21, J21, J24, J46, J53, M12, M14

Fecha de recepción: febrero 2 de 2017 / Fecha de aceptación: marzo 31 de 2017 / Publicado en línea: abril 19 de 2017 Tipología: Artículo de Investigación Científica y Tecnológica

Para citar este artículo: Sánchez, B. J., Viloria, E. J. \& Miranda, T. L. (2017). Los grupos informales en las organizaciones escolares: un acercamiento desde la gestión educativa. Praxis, 13(1), 56 - 68. Doi: http://dx.doi.org/10.21676/23897856.2068

1. Doctor en Educación. Docente de tiempo completo de la Universidad del Magdalena. Correo electrónico: joswaldosanchez@gmail.com ORCID: 0000-0002-9299-6647

2. Administrador de Empresas. Joven Investigador de la Universidad del Magdalena. Correo electrónico: javierviloriaescobar@gmail.com ORCID: 0000-0002-2396-4190

3. Estudiante de Administración de Empresas. Semillerista de Investigación de la Universidad del Magdalena. Correo electrónico: luisfranc17@gmail.com 


\section{INTRODUCCIÓN}

$E_{p}^{1}$ presente documento presenta resultados preliminares del proyecto de investigación "Prácticas de gestión directiva y académica que interpelan significativamente la calidad de las instituciones de educación básica y media del distrito de Santa Marta”, que desarrolla el Grupo de Análisis de la Cultura Escolar (GRACE), grupo de investigación que se encuentra en la Categoría A en Colciencias.

Esta investigación pretende comprender la realidad de las organizaciones que se dan en las instituciones educativas desde el paradigma emergente de su construcción social, basándose sobre todo en las investigaciones desarrolladas en el Doctorado en Ciencias de la Educación de la Universidad del Magdalena y, específicamente en la línea de Investigación, Administración y Desarrollo de los Sistemas Educativos. Así, el proyecto se configura como un espacio de reflexión sobre los componentes instituidos e instituyentes de las organizaciones escolares (Candamil, Murcia \& Sánchez, 2007) que inciden en la calidad del servicio educativo, haciendo mayor énfasis en los imaginarios que se construyen en la organización como resultado de las interacciones sociales y las aspiraciones de los actores educativos. Son estos aspectos los que le dan vida y sentido a las organizaciones, por lo que su gestión permite la generación de ventajas para las instituciones (Agudelo, Martínez \& Ortiz, 2013).

Los grupos informales son asociaciones espontáneas que emergen al interior de las organizaciones, en torno a objetivos e intereses comunes de las personas, y que no están expresados desde la configuración de una estructura formal. Estos grupos aparecerán cada vez que exista la organización legal debido a las necesidades de interacciones sociales de los actores. De esta forma, a nivel general, los grupos informales son construcciones sociales que responden a las necesidades y modos de pensamiento de las personas, no estando los centros educativos exceptos de su existencia.
Para abordar este fenómeno, se ha propuesto un sistema de categorías teóricas para la investigación, que fundamentan la comprensión conceptual y metodológica. Tales categorías son las siguientes:

\section{Categoría de encuadre 1. Componente psico-} social en las organizaciones educativas: $\mathrm{El}$ componente psicosocial de una organización se convierte en una fuente de ventajas competitivas y en un elemento de especial atención en la gestión directiva (Calderón, 2006). Es inevitable que, en las organizaciones, las personas desarrollen una tendencia a agruparse según los intereses comunes y las interacciones que se desprenden de las dinámicas funcionales de la estructura formal (Cequea \& Rodríguez, 2012).

Estas tendencias colectivas, que se desarrollan al interior de las organizaciones, generan un conjunto de dinámicas que influencian e impactan el funcionamiento organizacional, condicionando, a su vez, el comportamiento de las personas: se trata de tendencias que, por lo general, se dan a causa de una serie de patrones sociales que imperan en el contexto donde estas desarrollan y cumplen sus actividades cotidianas.

Tanto el componente psicosocial del factor humano, como la manera de gestionarlo, pueden constituir una fuente de ventaja competitiva sostenible (Álvarez, 2008; Gómez, 2014). En este sentido, dar importancia al componente humano en las organizaciones escolares implica una preocupación permanente por entender el comportamiento de las personas en el trabajo y los posibles efectos que este tiene sobre la estructura. El comportamiento grupal dentro de las organizaciones, en lo llamado "informal", debe generar puntos de análisis y reflexión por parte del sector educativo y motivar el aprovechamiento del mismo para beneficio de la organización escolar.

Categoría de encuadre 2. Construcción social de las organizaciones educativas: La construcción social de las organizaciones, como un paradigma emergente en el campo de la gestión 
educativa, se considera una línea de pensamiento en desarrollo que se alimenta de los aportes de ramas científicas como la psicología, la administración, la sociología y la antropología. Este ámbito investigativo sirve como soporte fundamental de nuevas comprensiones en los procesos de planeación y organización de las entidades educativas.

En dicho aspecto se plantea el imperativo de comprender y abordar los centros escolares desde una concepción humanista (Sánchez, 2009):

Se trata de sentar bases para repensar la concepción de la organización educativa como fundamento para transformar su desarrollo desde un punto de vista emergente en el cual la organización educativa se asume como institución social, viva, auto-genética, creadora, dinámica, compleja... (p. 97).

Para comprender a las organizaciones como sistemas vivos que interactúan con otros sistemas y que se constituyen debido a las relaciones sociales que se experimentan en su interior, es indispensable considerar los imaginarios que se van construyendo y reconstruyendo en cada proceso que se realiza. En este sentido, tales imaginarios se pueden configurar desde dos perspectivas según Sánchez (2012): la primera, hace referencia al sentido legalista de la organización, es decir, a los sentidos que se han definido desde las normas y reglas que componen la estructura formal de las organizaciones educativas, y, la segunda, comprende los acuerdos sociales e informales que surgen de los imaginarios de los actores y que se convierten en hechos institucionales. Di Marco (2015) sostiene que es a través de la comprensión de la realidad y de sus experiencias que se genera conocimiento.

Categoría Central. Grupos Informales: Para Viloria, Pedraza, Cuesta \& Pérez (2016) "los grupos informales hacen parte de las variables psicosociales que se producen de manera espontánea al interior de la organización y generan un conjunto de dinámicas que afectan el comportamiento de los empleados" (p. 32). Por tanto, es inevitable que, en el desarrollo de sus actividades, las personas establezcan un conjunto de interacciones sociales relacionadas con el trabajo y sus implicaciones humanas.

$\mathrm{Al}$ entenderse los grupos informales como asociaciones humanas no prescritas por la estructura legal de la organización educativa, se entiende también que existen factores que dan origen a los mismos al interior de la institución.

Davis \& Newstrom (2003), Kinicki \& Kreitner (2004) y Robbins (2004) consideran que las relaciones de amistad y empatía entre las personas son algunas de las causas principales que ocasionan el surgimiento de los grupos informales, mientras que Marqués, Farrerons, Arias, \& Quiroga (2012) señalan los intereses comunes como una de las razones para su existencia. Por su parte, Chiavenato (2009) expresa que la proximidad de los cargos también es un elemento fundamental que sirve como soporte para la creación de asociaciones informales en el interior de las organizaciones.

Lo realmente cierto es que, siempre que exista una estructura legal en cualquier tipo de organización, emergerán los grupos informales como consecuencia de las necesidades sociales de las personas. Estos, tendrán una incidencia directa en el comportamiento de los empleados, el funcionamiento organizacional y los resultados obtenidos (Viloria, Daza \& Pérez, 2016).

\section{Categoría asociada 1. Dinámicas de los grupos} informales: La investigación "Aproximación teórica a los grupos informales desde la Administración y la Psicología: una mirada a sus dinámicas e influencias en las organizaciones", realizada por Viloria \& Mena (2015), permitió identificar una serie de dinámicas propias de la organización que se presentan y se desarrollan con gran fuerza al interior de los grupos informales y afectan el funcionamiento organizacional.

A partir de esa investigación, Viloria, Daza \& Pérez (2016) realizan una caracterización y profundización de tales dinámicas para explicar la influencia de los grupos informales en las organizaciones. En este sentido, los grupos informales sirven como escenarios de cooperación entre las 
personas para el desarrollo de sus actividades laborales y personales. Además, se convierten en fuentes de generación de conocimiento para las organizaciones debido a que en ellos se identifican y comparten mejores maneras de realizar los procesos y actividades laborales.

También es claro que los grupos informales, debido a su interés propiamente social, sirven como canales para la comunicación de las personas, donde la información organizacional puede fluir sin filtros ni conductos regulares. Por otro lado, los grupos informales son plataformas permanentes de generación de liderazgo informal; este aspecto se convierte en la herramienta más poderosa para influir en el comportamiento y posición de las personas respecto a los objetivos de cualquier tipo de organización.

Desde esta perspectiva, los grupos informales al interior de las organizaciones escolares se convierten en herramientas que influyen directamente en el funcionamiento de las actividades académicas y administrativas de las instituciones.

Categoría asociada 2. Calidad de las organizaciones educativas: El concepto de "calidad en la educación”, aunque se inserta en unos parámetros de subjetividad a partir de construcciones individuales y colectivas de los actores involucrados (Menin, 2013), es fundamental para medir el desarrollo de las políticas educativas que se aplican y el efecto de las prácticas de la gestión directiva en las organizaciones escolares.

La implementación de una cultura de mejoramiento continuo se constituye en un elemento vital, para el funcionamiento organizacional adecuado y productivo, en términos de resultados evaluables. Al respecto, Álvarez \& Topete (2004) expresan su opinión sosteniendo que:

Entre las características de las instituciones educativas de calidad se encuentra un ambiente de superación, corresponsabilidad, autoexigencia y búsqueda de la mejora y superación continuas, por parte de profesores, alumnos, directivos, personal administrativo, padres o tutores, y de la propia comunidad. (p.15)
Esto, buscando la articulación entre los centros educativos, la comunidad y los organismos estatales (López, 2014).

Para comprender las realidades sociales de las organizaciones educativas, es pertinente indagar por las dinámicas que se desprenden de los procesos psicosociales de los empleados, ya sea en calidad de docentes, directivos o personal de apoyo, y que se realizan de forma paralela al funcionamiento legalista establecido por la estructura formal: "visualizándola, a su vez, como un gran tejido interactivo que engloba un conjunto de redes sociales interconectadas, podemos ganar una nueva y más compleja comprensión de la dinámica humana y la forma en que se vincula con la funcional" (Hernández, 2003, p.337).

A partir de lo anterior, y asumiendo que las asociaciones informales que existen al interior de las instituciones escolares tienen una influencia en el comportamiento de la comunidad educativa e impactan en el funcionamiento de los procesos, se toma como punto de partida o supuesto general que la existencia y gestión de los grupos informales incide en la calidad de las organizaciones, desde una mirada de las prácticas asociadas a la gestión educativa. Por lo tanto, se plantean como preguntas problematizadoras, en este punto de avance de la investigación: ¿Cuáles son los grupos informales que existen en las instituciones educativas de básica y media de carácter público del distrito de Santa Marta?, ¿por qué se originan dichos grupos informales?, y ¿cómo puede incidir la existencia de los grupos informales en la calidad de las instituciones educativas de básica y media de carácter público del distrito de Santa Marta?

\section{METODOLOGÍA}

\section{Tipo de investigación}

La investigación se asume y desarrolla principalmente con un enfoque cualitativo en el sentido planteado por Deslauriers (2004), cuando sostiene que las investigaciones cualitativas no desconocen las variables numéricas de la información sino que se enfocan en una comprensión 
de los procesos asociados a la construcción social de la realidad.

En este sentido, por un lado, la investigación se ubica en el plano de la intencionalidad básica debido a que pretende aportar a la comprensión y potenciación epistémica del paradigma emergente de la construcción social de las organizaciones a partir de la comprensión de la realidad de los grupos simbólicos o informales (Sánchez, 2014). Y, por otro lado, se trata de una investigación con un carácter propiamente aplicativo en la medida que busca, a partir de las configuraciones teóricas que se evidencien en el sentido básico, aportar a la transformación social de las organizaciones educativas entendidas como sistemas vivos.

En un contexto general, la investigación se moviliza en el sentido comprensión-transformación, permitiendo que también tenga una dinámica de desarrollo teórico-desarrollo social, como consecuencia de las triangulaciones que se presentan entre los datos, investigadores, aspectos teóricos y metodológicos.

Los trayectos de la investigación se abordan desde la perspectiva de la complementariedad, asumiendo que en una investigación de este tipo "las estructuras se van descubriendo o configurando progresivamente, a medida que se interactúa con el fenómeno sujeto de estudio" (Murcia \& Jaramillo, 2000, p. 95).

A partir de lo anterior, la investigación permite abordar el proceso investigativo desde diversas posturas metodológicas y complementar los instrumentos de recolección de información.

\section{Unidad de análisis y actores sociales}

En su ámbito general, la investigación tiene como unidades de análisis o rangos poblacionales las instituciones educativas de básica y media de carácter público del distrito de Santa Marta; sin embargo, en este documento se recoge un ejercicio etnográfico realizado en una institución en particular donde se ha evidenciado la existencia de diversos factores sociales que han propiciado el surgimiento de grupos informales con alta incidencia en los procesos asociados a la gestión educativa. Para esto, se han tomado como actores sociales de análisis a los docentes, directivos, estudiantes y personal de apoyo vinculados a las instituciones.

\section{Instrumentos de recolección de información}

Hasta este punto de la investigación se han utilizado como instrumentos para la recolección de la información:

Análisis documental: asumido desde Peña \& Pirela (2007), consiste en una revisión profunda de las normas, manuales y principios organizacionales que se han establecido en la organización educativa para encuadrar el horizonte institucional y el funcionamiento de los procesos de gestión.

Diario de campo: es una herramienta de investigación (Jiménez, 2011) que ha permitido el registro de actividades y experiencias vividas en la organización donde se evidencia alguna dinámica asociada a los grupos informales. En el diario se hace un registro de la experiencia y se propone una comprensión inicial de parte de los investigadores.

Observación participante: Angrosino (2012) la entiende como una herramienta de investigación cualitativa que se asume como un proceso que permite hacer un seguimiento de los componentes sociales en la organización, promoviendo la vinculación de los investigadores a los procesos de gestión y a las actividades cotidianas de la institución.

Entrevistas en profundidad: consisten en un diálogo semiestructurado entre los investigadores y las personas que se han identificado como participantes de un grupo informal con el fin de conocer las dinámicas propias de dicho grupo y los intereses por los cuales se formó al interior de la institución.

\section{Procedimiento}

La investigación se ha configurado de una manera muy dinámica y flexible, permitiendo que se 
desarrollen los procesos desde una perspectiva complementaria y que se configuren los momentos a medida que se profundiza en el fenómeno. En este sentido, se hace una preconfiguración de la realidad, una configuración de la realidad y, por último, una reconfiguración de la realidad.

Los momentos mencionados se desarrollan durante tres etapas centrales de la investigación:

1. Identificación de los grupos informales en la organización educativa.

2. Comprensión de las razones que originan los grupos informales en la organización educativa.

3. Interpelación de aspectos positivos y negativos de los grupos informales en la organización educativa.

A partir de tales consideraciones se realiza la recolección y análisis de la información y luego se presentan los resultados hasta este punto de la investigación.

\section{RESULTADOS Y DISCUSIÓN}

En este punto se presentan resultados preliminares de la investigación a partir de los objetivos que se han definido. Lo que se pretende es explorar la existencia de los grupos informales en las organizaciones escolares, por lo que inicialmente se presenta una caracterización de los grupos informales que se han identificado en la unidad focal de estudio: luego, se enlistan algunos de los factores que han contribuido al origen y existencia de tales grupos informales, y, por último, se proponen algunas influencias de los colectivos sociales informales en el funcionamiento y calidad de las organizaciones educativas.

\section{Identificación de Grupos Informales en las Organizaciones Educativas}

Considerando que se ha definido una unidad de análisis específica para el estudio de los grupos informales, se han logrado identificar diversos tipos que se nombran en relación a las actividades alrededor de las cuales se constituyen. Estos grupos se codifican de la siguiente manera: G (que significa Grupo), I (que equivale a Informal), y C (una constante para enumerar el grupo). En la tabla 1, que se encuentra a continuación, se relacionan los hallazgos, se les otorga una denominación coloquial y se hace una descripción de los grupos informales identificados.

Tabla 1: Grupos informales en la institución

\begin{tabular}{|l|l|l|}
\hline Código & Denominación & \multicolumn{1}{c|}{ Descripción } \\
\hline GI-1 & "Los futbolistas" & $\begin{array}{l}\text { Lo constituyen docentes y personal administrativo que participa en ac- } \\
\text { tividades deportivas asociadas al futbol. Este grupo informal se reúne } \\
\text { constantemente para la planificación de sus actividades, para los entrena- } \\
\text { mientos y para compartir opiniones en torno a las dinámicas del deporte } \\
\text { en el plano nacional e internacional. Los miembros de este gurpo son } \\
\text { muy cercanos a los estudiantes debido a que confluyen en las actividades } \\
\text { recreativas de la institución y desarrollan encuentros deportivos con ellos. }\end{array}$ \\
\hline GI-2 & $\begin{array}{l}\text { "Lstá conformado por un número reducido de docentes y directivos que tienen } \\
\text { un alto grado de acercamiento al rector de la institución y que defienden y } \\
\text { promueven sus iniciativas en la comunidad académica. Este grupo informal } \\
\text { goza de un alto nivel de influencia en la institución, siendo sus posturas } \\
\text { siempre respaldadas por la alta dirección. Es un grupo muy cerrado al que } \\
\text { pocas personas pueden tener acceso. }\end{array}$ \\
\hline GI-3 & $\begin{array}{l}\text { Es un grupo informal que promueve y organiza diversos eventos académicos, } \\
\text { culturales y deportivos al interior de la institución. Lo integran docentes y } \\
\text { estudiantes de grados superiores que convocan y movilizan a la comunidad } \\
\text { académica en torno a los eventos que se planifican. }\end{array}$ \\
\hline
\end{tabular}




\begin{tabular}{|c|c|c|}
\hline Código & Denominación & Descripción \\
\hline GI-4 & "Los políticos" & $\begin{array}{l}\text { Es un grupo informal de docentes y directivos que comparten posiciones } \\
\text { políticas diferentes y se reúnen para debatir sobre temas de actualidad nacio- } \\
\text { nal e internacional. Se caracterizan en que se reúnen a dialogar compartir } \\
\text { en espacios libres, como lo son las áreas de cafetería y las salas docentes. }\end{array}$ \\
\hline GI-5 & $\begin{array}{l}\text { "Los } \\
\text { inconformes" }\end{array}$ & $\begin{array}{l}\text { Es un grupo informal compuesto por docentes que consideran que la gestión } \\
\text { de la institución es insuficiente con relación a los retos y necesidades de la } \\
\text { comunidad estudiantil. Tienen una posición contraria a la administración } \\
\text { de la institución y, en su conjunto, son docentes que tienen una amplia } \\
\text { trayectoria al interior de la escuela y que se sienten relegados por personas } \\
\text { de menor edad en la dirección y coordinación de procesos fundamentales. }\end{array}$ \\
\hline GI-6 & $\begin{array}{l}\text { "Los de servicios } \\
\text { generales" }\end{array}$ & $\begin{array}{l}\text { Es un grupo informal conformado por las personas que hacen parte de la } \\
\text { nómica de apoyo de la institución. En él, comparten sus intereses, preocu- } \\
\text { paciones y necesidades, siendo un espacio para descargar sus experiencias } \\
\text { al interior de la organización, así como para presentar sus opiniones sobre } \\
\text { la gestión, los procesos y el personal de la institución. }\end{array}$ \\
\hline GI-7 & $\begin{array}{l}\text { "Los } \\
\text { informantes" }\end{array}$ & $\begin{array}{l}\text { Es un grupo informal conformado por docentes, directivos y personal de } \\
\text { apoyo que se dedica a compartir información sobre las demás personas } \\
\text { integrantes de la comunidad académica. En su interior, se conocen todos } \\
\text { los chismes de la institución y se maneja la información personal, familiar } \\
\text { y laboral de los principales personajes de la misma. }\end{array}$ \\
\hline GI-8 & $\begin{array}{l}\text { "Los líderes } \\
\text { académicos" }\end{array}$ & $\begin{array}{l}\text { Es un grupo integrado por docentes y estudiantes que presta especial } \\
\text { atención a los asuntos académicos de la institución. Este grupo se dedica } \\
\text { principalmente a compartir innovaciones y conocimientos que se generan } \\
\text { en los campos científicos donde cada uno labora. Así también, los miembros } \\
\text { de este grupo son los encargados de organizar y liderar los procesos acadé- } \\
\text { micos de la institución y de promocionarlos en la comunidad académica. }\end{array}$ \\
\hline GI-9 & "Los fiesteros" & $\begin{array}{l}\text { Está constituido por docentes, directivos y personal de apoyo de la ins- } \\
\text { titución, quienes se dedican principalmente a organizar actividades de } \\
\text { entrenamiento e integración de la comunidad educativa y a convocar a } \\
\text { los actores sociales para que participen en las mismas. Tienen un amplio } \\
\text { reconocimiento en la institución entre todos los estamentos. }\end{array}$ \\
\hline GI-10 & $\begin{array}{l}\text { "Los } \\
\text { comunitarios" }\end{array}$ & $\begin{array}{l}\text { Es un grupo pequeño conformado por docentes y estudiantes que busca } \\
\text { la articulación de la institución con la comunidad. Se enfoca en la organi- } \\
\text { zación y desarrollo de actividades asociadas a la gestión comunitaria y, en } \\
\text { especial, en la estimulación de la participación de diversos actores en las } \\
\text { actividades propias de la escuela. }\end{array}$ \\
\hline
\end{tabular}

Fuente: elaboración propia a partir de información recolectada

Estos grupos, que se han reconocido hasta este punto en la organización escolar, tienen sus propias dinámicas y alcances; es decir permean e inciden en los procesos organizacionales de la escuela. Es importante resaltar que algunas personas participan en dos o más grupos informales y que se han identificado los líderes más influyentes de cada uno de ellos. Además, los niveles de cohesión de los diferentes grupos son lo suficientemente altos como para garantizar su supervivencia en el tiempo al interior de la organización.

\section{Razones para el Surgimiento de Grupos Informales en las Organizaciones Educativas}

A partir de las instancias dialógicas que se han implementado en la investigación, se han logrado recuperar, desde las voces de los actores sociales, algunas de las razones o factores por los cuales se originan los grupos informales en las organizaciones educativas. 
En este sentido, se evidencia que entre las causas más comunes están las relaciones de amistad entre los integrantes debido a la existencia de sentimientos y experiencias históricas compartidas: "Creo que uno se reúne de manera frecuente con algunas personas en específico debido a que existen relaciones personales de amistad que se han ido construyendo durante todo el tiempo que se comparte en la institución" (Actor Educativo). Al respecto, Martínez (2013) expresa que en los escenarios educativos se desarrollan un conjunto de dinámicas sociales entre los actores como consecuencia de las interacciones y las prácticas pedagógicas.

Además, también se reconocen como factores determinantes en la creación de grupos informales la proximidad en los cargos y en las funciones desempeñadas, las interacciones laborales al interior de la organización, los intereses comunes de las personas en cuanto a ideologías políticas, y la posición de las mismas frente a la dirección institucional.

Las relaciones personales con algunas personas están mediadas y originadas por muchas razones. En mi caso, por ejemplo, tengo en cuenta lo que piensan los demás con respeto al funcionamiento de los procesos de la escuela. Aquí muchas cosas han empezado a funcionar de manera negativa y es importante que los colectivos tomen consciencia de esta realidad para promover procesos de cambio. (Actor Educativo)

La evaluación de los procesos y de los actores educativos en los centros escolares permite realizar valoraciones y establecer estrategias para el mejoramiento de la calidad de la institución (Maussa, 2014), si bien en muchos casos los actores de algunas organizaciones rechazan los modelos institucionales ocasionando conflictos (Ureña, Jiménez, Mejía \& Vilarete, 2014).

Por otro lado, criterios relacionados con las perspectivas deportivas, culturales y académicas también inciden en el surgimiento de los grupos dentro de la institución, debido a que las personas eligen como factores de agrupamiento las dinámicas de sus compañeros con respecto a estos elementos que les permiten establecer una relación entre la escuela y la comunidad para la resignificación de las prácticas pedagógicas de la escuela (Villa \& Villa, 2014).

Definitivamente uno se siente mejor con personas con las que desarrolla actividades comunes como la participación en eventos deportivos, pedagógicos, comunitarios, culturales o académicos. Siente uno que se existe una mejor comunicación y que se disfrutan de mejor manera los momentos que se comparten. Es muy difícil, por ejemplo, que si a me gusta el futbol me sienta más cómodo con las personas que disfrutan bailar o las actividades artísticas. (Actor Educativo)

Por otro lado, existen elementos que, aunque con menor fuerza, también inciden en el surgimiento de los grupos informales en la institución educativa. Entre estos se encuentran las aspiraciones o sueños profesionales, las necesidades sociales y las creencias religiosas. Variables que están relacionadas con las expectativas de las personas de superar las condiciones sociales y económicas del contexto local y mundial (Balza, 2013).

Todos estos factores han propiciado y permitido la supervivencia de los grupos informales a partir de los niveles de cohesión que se han consolidado como consecuencia de la intensidad de los intereses compartidos por los integrantes del colectivo informal.

Lo más importante para permanecer en un grupo de personas en especial que no es propio de las actividades laborales en la institución, son los niveles de armonía que existan en ese grupo. Es necesario que todos tengan buenas relaciones y que siempre se priorice el sentido o interés por el que existe el equipo. (Actor Educativo)

\section{Posibles Influencias de los Grupos Informales en la Calidad de las Organizaciones Educativas}

Al entender las organizaciones educativas como sistemas vivos que se configuran por la interacción y confluencia de las personas, se denotan una serie de posibles incidencias, tanto positivas como negativas, que se desprenden de la existencia de los grupos informales. 
En este sentido, si un determinado grupo informal toma una posición contra-organizacional puede, bien incidir negativamente en los resultados de las evaluaciones que se realizan a la institución, bien afectar el buen funcionamiento de los procedimientos. Por ejemplo, si el grupo informal que se dedica a la articulación de la escuela con la comunidad decide no seguir estimulando dichas actividades, la organización disminuirá sus indicadores en la evaluación de la gestión comunitaria. Mientras que si el grupo continúa con la promoción de las estrategias de participación comunitaria se fortalecerá el vínculo entre sociedad y escuela.

Es claro que los rendimientos de la institución en gestión comunitaria estarán determinados en gran medida por las actividades que desarrollamos las personas a las que nos interesa el tema. Si nosotros no impulsamos estas actividades, otras personas en la escuela no lo harán y habrá una desarticulación entre la comunidad y la institución. (Actor Educativo)

Para ilustrar otra situación en la escuela veamos esto: si el grupo informal que se formó para la comunicación de los chismes de los actores educativos toma una posición de mayor divulgación de la información que manejan en su interior, la imagen de algunos personajes que hacen parte de la vida cotidiana de la organización se verá perjudicada, generándose conflictos en la organización.

En la tabla 2, que se encuentra a continuación, se presentan las posibles influencias de cada grupo informal identificado en los procesos de gestión educativa de la institución y en los niveles de calidad de la misma. Estas relaciones se deducen de las voces de los actores educativos.

Tabla 2: influencias de los grupos informales en la institución

\begin{tabular}{|c|c|c|}
\hline $\begin{array}{c}\text { Grupo } \\
\text { informal }\end{array}$ & Posibles incidencias negativas & Posibles incidencias positivas \\
\hline GI-1 & $\begin{array}{l}\text { Afectaciones en los tiempos de la jornada escolar. } \\
\text { Descuido de las actividades académicas. } \\
\text { Inasistencia a las actividades académicas. }\end{array}$ & $\begin{array}{l}\text { Aportes al bienestar del talento humano en la } \\
\text { organización. } \\
\text { Participación de la comunidad en las actividades } \\
\text { de la escuela. } \\
\text { Generación de actividades extracurriculares. } \\
\text { Aprendizaje de estrategias para el trabajo en } \\
\text { equipo. } \\
\text { Fortalecimiento de las actividades de integración } \\
\text { entre los actores sociales. }\end{array}$ \\
\hline GI-2 & $\begin{array}{l}\text { Sesgos en las planificaciones y asignaciones } \\
\text { académicas. } \\
\text { Flexibilidad en el seguimiento a las actividades en } \\
\text { el aula. } \\
\text { Desconocimiento de los sistemas de gobierno } \\
\text { escolar. } \\
\text { Malestares en la cultura institucional. }\end{array}$ & $\begin{array}{l}\text { Agilización de los procesos y trámites } \\
\text { institucionales. } \\
\text { Respaldo al liderazgo directivo de la institución. } \\
\text { Descentralización de procesos institucionales. }\end{array}$ \\
\hline GI-3 & $\begin{array}{l}\text { Modificación de las jornadas escolares. } \\
\text { Necesidades de acomodación de los espacios } \\
\text { físicos. } \\
\text { Requerimiento de asignación de recursos financie- } \\
\text { ros de la institución. }\end{array}$ & $\begin{array}{l}\text { Fortalecimiento de la planificación estratégica } \\
\text { institucional. } \\
\text { Promoción de la integración de los actores } \\
\text { educativos. } \\
\text { Contribución a la participación y convivencia. } \\
\text { Proyección de la escuela a la comunidad. } \\
\text { Promoción de recursos para el aprendizaje. } \\
\text { Motivación a los actores educativos hacia el } \\
\text { aprendizaje. }\end{array}$ \\
\hline
\end{tabular}




\begin{tabular}{|c|c|c|}
\hline $\begin{array}{l}\text { Grupo } \\
\text { informal }\end{array}$ & Posibles incidencias negativas & Posibles incidencias positivas \\
\hline GI-4 & $\begin{array}{l}\text { Posibilidad de redefinir el horizonte estratégico. } \\
\text { Modificación de las políticas de la escuela. } \\
\text { Influencia en las decisiones de los órganos de } \\
\text { dirección. } \\
\text { Incidencia en las opiniones de las autoridades } \\
\text { educativas. }\end{array}$ & $\begin{array}{l}\text { Reconocimiento de las instancias del gobierno } \\
\text { escolar. } \\
\text { Expresión de la necesidad de la interacción polí- } \\
\text { tica con el sector. } \\
\text { Exigencia de rendición de cuentas. } \\
\text { Aportación al direccionamiento estratégico y al } \\
\text { horizonte institucional. }\end{array}$ \\
\hline GI-5 & $\begin{array}{l}\text { Oposición al direccionamiento estratégico. } \\
\text { Críticas a la gestión estratégica y el gobierno } \\
\text { escolar. } \\
\text { Deslegitimación de las acciones institucionales. } \\
\text { Alteración del buen funcionamiento del clima } \\
\text { escolar. } \\
\text { Afectación de la imagen de las instituciones ante la } \\
\text { comunidad. } \\
\text { Criticas a los planes y modelos académicos. }\end{array}$ & $\begin{array}{l}\text { Ayudas a la evaluación, seguimiento y rendición } \\
\text { de cuentas en la institución. } \\
\text { Fortalecimiento de las instancias del gobierno } \\
\text { escolar. } \\
\text { Promoción de la vinculación de la comunidad en } \\
\text { la escuela. } \\
\text { Vigilancia de las prácticas pedagógicas, de la } \\
\text { gestión del aula y del seguimiento académico. } \\
\text { Contribución a la promoción de buenas prácticas } \\
\text { de gestión educativa. }\end{array}$ \\
\hline GI-6 & $\begin{array}{l}\text { Distorsión delos canales y calidad de la } \\
\text { comunicación. } \\
\text { Posibilidad de que se ignoren los programas de } \\
\text { seguridad. } \\
\text { Modificación del ambiente físico. }\end{array}$ & $\begin{array}{l}\text { Fortalecimiento del trabajo en equipo. } \\
\text { Conservación de los recursos para el } \\
\text { aprendizaje. } \\
\text { Mantenimiento, adecuación y embellecimiento } \\
\text { de la planta física. } \\
\text { Contribución a la gestión responsable de la } \\
\text { institución. }\end{array}$ \\
\hline GI-7 & $\begin{array}{l}\text { Generación de malestares en el ambiente y cultura } \\
\text { escolar. } \\
\text { Modificación de la información del personal } \\
\text { institucional. } \\
\text { Afectación de la imagen de la escuela ante la } \\
\text { comunidad. } \\
\text { Producción de conflictos en la convivencia } \\
\text { institucional. }\end{array}$ & $\begin{array}{l}\text { Contribución a la creación y ajuste de normas } \\
\text { para la convivencia institucional. } \\
\text { Reconocimiento educativo y social de los actores } \\
\text { sociales. }\end{array}$ \\
\hline GI-8 & Modificación de la planificación académica. & $\begin{array}{l}\text { Introducción de nuevos conceptos en la gestión } \\
\text { académica. } \\
\text { Aportación a la resignificación pedagógica. } \\
\text { Fortalecimiento del seguimiento y de la evalua- } \\
\text { ción académica. } \\
\text { Promoción de nuevas opciones didácticas para } \\
\text { mejorar el aprendizaje. }\end{array}$ \\
\hline GI-9 & $\begin{array}{l}\text { Disminución del rendimiento académico y laboral } \\
\text { de los actores educativos. } \\
\text { Generación de irresponsabilidades en la comunidad } \\
\text { académica. }\end{array}$ & $\begin{array}{l}\text { Aumento de los niveles de integración social en } \\
\text { los actores educativos. } \\
\text { Fortalecimiento del talento humano. } \\
\text { Incremento de la motivación laboral. }\end{array}$ \\
\hline GI-10 & $\begin{array}{l}\text { Posible distorsión de la imagen de la escuela en la } \\
\text { comunidad. }\end{array}$ & $\begin{array}{l}\text { Contribución a la inclusión social en la escuela. } \\
\text { Dinamización de las relaciones con el entorno. } \\
\text { Vinculación de la comunidad con los órganos } \\
\text { colegiados de la institución. } \\
\text { Fortalecimiento de las relaciones con el sector } \\
\text { productivo, con otras instituciones y con las } \\
\text { autoridades locales. } \\
\text { Respaldo a la diversidad cultural. }\end{array}$ \\
\hline
\end{tabular}

Fuente: elaboración propia a partir de información recolectada. 
En este sentido, depende de la posición a favor o en contra que tomen los grupos informales en la organización, los impactos positivos o negativos que se den en la calidad educativa; debido a que, como se vio, las dinámicas propias de los grupos pueden afectar los procesos y áreas de la gestión educativa.

\section{CONCLUSIONES}

El componente humano de las organizaciones educativas complejiza y determina el buen funcionamiento organizacional y dota de determinados simbolismos informales las experiencias vividas por los trabajadores.

Desde esta perspectiva, los componentes psicosociales de los empleados en las organizaciones condicionan la generación de acercamientos y asociaciones en el trabajo; por lo tanto, es inevitable que surjan los grupos informales como respuesta a las necesidades sociales de los actores sociales participantes de los procesos de gestión en la escuela.

Los grupos informales emergen entonces como escenarios de confluencia de las personas y se convierten en plataformas de condicionamiento social en el trabajo y de incidencia en la calidad de las organizaciones educativas. En este punto, si un grupo informal toma una posición contra-organizacional afectará la calidad de la institución en las mediciones que realicen los organismos competentes y se desencadenarán una serie de dinámicas que pueden generar conflictos al interior de la organización.

De esta manera, el reconocimiento de los grupos informales en las instituciones educativas es de trascendental importancia para el desarrollo de una gestión educativa que dé respuesta al complejo entramado psicosocial que define a este tipo de organizaciones. Así las cosas, el conocimiento sobre los grupos informales existentes y la comprensión de sus dinámicas propiciarán una nueva visión de la gestión educativa en las instituciones, caracterizada por la promoción de nuevas alternativas para un liderazgo de gestión en las mismas.
La gestión directiva, la gestión académica, la gestión administrativa y financiera, y la gestión comunitaria son áreas de la gestión educativa que se ven interpeladas de manera positiva y negativa por la presencia de los grupos informales. Cada uno de los procesos que integran estas áreas recibe influencias directas o indirectas como consecuencia de las dinámicas y el funcionamiento de los colectivos sociales.

Es importante, por lo tanto, tener un conocimiento académico pertinente sobre los grupos informales y sus dinámicas al interior de las organizaciones educativas, puesto que de esta forma sería posible, por un lado, explotar sus alcances a favor de las metas y horizontes institucionales. Y, por otro lado, crear redes sociales poderosas al interior de la escuela que permitan las construcciones sociales de la realidad educativa y privilegien los imaginarios sociales que se configuran tanto al interior de las normas y reglas de la institución, como en torno a las aspiraciones y metas de los actores sociales.

A partir de estos resultados, se deduce que es recomendable profundizar en las dinámicas propias de los grupos informales y en sus incidencias en la calidad de las organizaciones educativas para desarrollar herramientas que permitan gestionar sus potencialidades en beneficio de los objetivos organizacionales. En este sentido, una dinámica de especial interés investigativo y comprensivo es el liderazgo informal al interior de los grupos informales, en la medida de que se convierte en la principal herramienta de influencia en el comportamiento y posición de los empleados con relación a los intereses de los mismos.

Por tanto, es fundamental abordar, desde el plano académico e investigativo, la gestión de los grupos informales en las organizaciones escolares; así, se podrá contar con herramientas que permitan potenciar las bondades del capital humano en perspectiva del mejoramiento de la calidad institucional de la escuela mediante el diseño, desarrollo y evaluación de procesos de intervención en las estructuras formal e informal, y se conseguirá alcanzar una configuración 
global de la gestión de los centros educativos. Sólo logrado este punto será posible comprender el valioso aporte de los grupos informales a la gestión estratégica de las instituciones educativas y al cumplimiento de su misión institucional.

\section{REFERENCIAS BIBLIOGRÁFICAS}

Agudelo, C., Martínez, L., \& Ortiz, I. (2013). Gestión del conocimiento: un activo intangible a través de la investigación. Praxis, 9, 108-113. Recuperado de http://revistas.unimagdalena.edu.co/index.php/praxis/article/ view/750/694

Álvarez, A. (2008). Gerencia del Talento Humano: Programa de Administración Pública Territorial. Bogotá. D.C.: Escuela Superior de Administración Pública.

Álvarez, I., \& Topete, C. (2004). Búsqueda de la calidad en la educación básica. Conceptos básicos, criterios de evaluación y estrategia de gestión. Revista Latinoamericana de Estudios Educativos (México), XXXIV(3), 1136. Recuperado de http://www.redalyc.org/ articulo.oa? $\mathrm{id}=27034302$

Angrosino, M. (2012). Etnografía y observación participante en investigación cualitativa. Madrid: Ediciones Morata.

Balza, V. (2013). La espiral viciosa de la pobreza. Clío América, 7(14), 170-176. Recuperado de http://revistas.unimagdalena.edu.co/index. $\mathrm{php} /$ clioamerica/article/view/761

Calderón, G. (2006). La Gestión Humana y sus aportes a las organizaciones colombianas. Cuadernos de Administración, 19(31), 9-55. Recuperado de http://www.redalyc.org/ pdf/205/20503102

Candamil, M., Murcia, N., \& Sánchez, J. (2007). Autoevaluación, Acreditación y Vida Universitaria (Proyecto de investigación). Manizales: Universidad de Caldas.

Cequea, M., \& Rodríguez-Monroy, C. (2012). Productividad y Factores Humanos. Un modelo con ecuaciones estructurales. Interciencia, 37(2), 121-127. Recuperado de http://www. redalyc.org/articulo.oa? id $=33922717007$

Chiavenato, I. (2009). Comportamiento Organizacional. La dinámica del éxito en las organizaciones. Colombia: Mc Graw Hill.
Davis, K. \& Newstrom, J. (2003). Comportamiento Humano en el trabajo. México D.F.: Mc Graw Hill. Deslauriers, J. (2004). Investigación Cualitativa: Guía Práctica. RUDECOLOMBIA. Pereira, Colombia: Ediciones Papiro.

Di Marco, R. (2015). En busca del origen del conocimiento: el dilema de la realidad. Praxis, 11, 150-162. Recuperado de http://revistas.unimagdalena.edu.co/index.php/praxis/article/ view/1561/999

Gómez, D. (2014). Apuntes desde la ciencia económica ¿el sujeto racional o el sujeto complejo? Clío América, 8(15), 83-89. Recuperado de http://revistas.unimagdalena.edu.co/index. php/clioamerica/article/view/831/756

Hernández, A. (2003). Informalidad Organizacional y Redes. Convergencia. Revista de Ciencias Sociales, 10(32), 337-358. Recuperado de http://convergencia.uaemex. $\mathrm{mx} /$ article/view/1630

Jiménez, D. J. (2011). El diario como un instrumento de autoformación e investigación. Qurriculum: Revista de teoría, investigación y práctica educativa, 24, 173-200.

Kinicki, A., \& Kreitner, R. (2004). Comportamiento Organizacional: Conceptos, problemas y prácticas. México D.F.: McGraw-Hill Interamericana Editores S.A. de C.V.

López, J. (2014). Modelos interpretativos de la relación estado-empresa-universidad. Clío América, 8(15), 111-122. Recuperado de http:// revistas.unimagdalena.edu.co/index.php/ clioamerica/article/view/833

Martínez, A. (2013). Emergencias de cambio: entre el modelo pedagógico tradicional y la necesidad de aprendizajes significativos. Praxis, 9, 73-82. Recuperado de http://revistas.unimagdalena.edu.co/index.php/praxis/article/ view/746/690

Marqués, P., Farrerons, L., Arias, N. \& Quiroga, E. (2012). Las relaciones informales: un valor añadido en la gestión de personas. Revista electrónica trimestral de Enfermería, 26, 310-323. Recuperado de http://revistas.um.es/eglobal/ article/view/eglobal.11.2.139101/133601

Maussa, E. (2014). Características, principios y fines de la evaluación del desempeño docente. Praxis, 10, 8-20. Recuperado de http://revistas.unimagdalena.edu.co/index.php/praxis/ article/view/1355 
Menin, O. (2013). ¿Qué es una educación de calidad? Praxis Educativa (Arg), 17(1), 24-27. Recuperado de http://ojs.fchst.unlpam.edu. ar/ojs/index.php/praxis/article/view/579

Murcia, N., \& Jaramillo, L. (2000). La investigación cualitativa. La investigación etnográfica. Armenia: Kinesis.

Peña, T., \& Pirela, J. (2007). La complejidad del análisis documental. Información, cultura y sociedad, (16), 55-81. Recuperado de http:// www.scielo.org.ar/scielo.php? script $=$ sci arttext\&pid $=$ S1851-17402007000100004\&lng $=$ es\&tlng $=\mathrm{es}$

Robbins, S. (2004). Comportamiento Organizacional. México D.F.: Pearson Educación.

Sánchez, J. (2009). Una perspectiva emergente para concebir la organización educativa como fundamento para transformar su desarrollo. En J. Caro. (Ed.), Cultura Y Sociedad: Orientaciones Para El Ser Humano En Las Organizaciones. (pp.191-211). Santa Marta, Colombia: Editorial Unimagdalena.

Sánchez, J. (2012). La constitución del objeto de estudio de la Linea de Investigación sobre Administración y Desarrollo de los Sistemas Educativos. Doctorado en Ciencias de la Educación Universidad del Magdalena - RUDECOLOMBIA. Santa Marta, Colombia.

Sánchez, J. (2014). Prácticas de Autoevaluación con fines de Acreditación Institucional en Colombia: una lectura evaluativa y propositiva desde la perspectiva de la construcción social de la realidad. Santa Marta: Editorial Unimagdalena.
Ureña, Y., Jiménez, I., Mejía, A., \& Vilarete, J. (2014). Cultura innovativa: prospectiva de las condiciones estratégicas para el desarrollo del capital humano. Clío América, 8(16), 195-200. Recuperado de http://revistas.unimagdalena.edu.co/index.php/clioamerica/article/ view/1353

Villa, E., \& Villa, W. (2014). Los saberes de la negación y las prácticas de afirmación una vía para la pedagogización desde una perspectiva otra en la escuela. Praxis, 10, 21-36. Recuperado de http://revistas.unimagdalena. edu.co/index.php/praxis/article/view/1356

Viloria, J., \& Mena, A. (2015). Aproximación teórica a los grupos informales desde la Administración y la Psicología: una mirada hacia sus dinámicas e influencias en la organización. (Tesis de pregrado). Universidad del Magdalena, Santa Marta.

Viloria, J., Daza, A., \& Pérez, K. (2016). Dinámicas e influencias de los grupos informales en las organizaciones. Anfora, 23(40), 169-194. Recuperado de http://publicaciones. autonoma.edu.co/index.php/anfora/article/ view/9/7

Viloria, E. J., Pedraza, A. L., Cuesta, T. K., \& Pérez, C. K. (2016). Liderazgo informal en las organizaciones: reflexiones sobre su impacto e influencia en la competitividad. Clío América, 10(19), 31-42. Recuperado de http://revistas. unimagdalena.edu.co/index.php/clioamerica/ article/view/1658/1118 